\title{
Endocarditis trombótica no bacteriana y adenocarcinoma de endometrio
}

\author{
Jorge Casal-Lorenzo ${ }^{1}$, César Barbolla-Sancho ${ }^{1}$, Paula Gargallo-Fernández² ${ }^{2}$ Manuel Jesús Núñez-Fernández \\ ${ }^{1}$ Servicio de Medicina Interna. Complejo Hospitalario de Pontevedra. ${ }^{2}$ Servicio de Cardiología. Complejo Hospitalario de Pontevedra.
}

\section{RESUMEN}

Se describe el caso de una mujer de 80 años, con antecedentes de trombosis venosa profunda en tratamiento con apixaban, que presenta clínica focal neurológica. Las pruebas de imagen evidencian múltiples émbolos arteriales en cerebro, riñones y bazo, así como imagen sugestiva de neoplasia uterina. Una biopsia tras histeroscopia, confirma el diagnóstico de adenocarcinoma de endometrio. La ecocardiografía transesofágica constata la existencia de vegetaciones en válvulas cardíacas, siendo los estudios infecciosos negativos. Es diagnosticada de endocarditis trombótica no bacteriana relaciona con una neoplasia. Se cambia apixaban por heparina de bajo peso molecular, confirmando la práctica desaparición de las vegetaciones.

Palabras clave: endocarditis trombótica no bacteriana, endocarditis marántica, adenocarcinoma endometrial, trombosis venosa profunda, apixaban.

\section{INTRODUCCIÓN}

La relación entre cáncer y trombosis se establece por primera vez en 1866 por Trousseau, y desde entonces han sido publicados multitud de trabajos confirmando dicha asociación. La endocarditis trombótica no bacteriana (ETNB) aparece en situaciones de hipercoagulabilidad, entre las que se encuentra el cáncer. Los tumores que más frecuentemente se asocian con ETNB son los adenocarcinomas localizados en pulmón, páncreas, estómago y ovario¹.

La descripción de ETNB y cáncer de útero no es frecuente, sólo han sido publicados 9 casos $^{2-10}$ (en tres de forma simultánea con cáncer de ovario). Se expone un nuevo caso de cáncer de útero presentándose inicialmente con una trombosis venosa profunda (TVP) y una ETNB, con la particularidad de que recibió tratamiento con apixaban antes de presentar fenómenos embólicos arteriales.

\section{CASO CLÍNICO}

Mujer de 80 años, ingresada por síndrome confusional en probable relación con infección urinaria y respiratoria. El día del ingreso acude al Servicio de Urgencias por desorientación, tos, disnea, escasa expectoración amarillenta, sin dolor torácico, ni fiebre.

Vive con su familia, con funciones cerebrales superiores adecuadas, siendo independiente para todas las funciones, deambulando con dificultad por artrosis. Tiene antecedentes de hipertensión arterial, dislipemia e insuficiencia venosa crónica con episodio previo de úlcera varicosa resuelto.

Un mes antes de su ingreso es diagnosticada de una TVP en vena femoral superficial derecha. Recibe tratamiento con apixaban a dosis de $5 \mathrm{mg}$ cada 12 horas desde entonces;

\begin{abstract}
We present a case of an 80-year-old woman with a history of deep vein thrombosis, treated with apixaban, which has a focal neurological clinic. Imaging tests show multiple arterial ischemic lesions in brain, kidneys and spleen, as well as suggestive imaging of uterine neoplasia. A biopsy after hysteroscopy confirms the diagnosis of endometrial adenocarcinoma. Transesophageal echocardiography shows vegetations in valves, with negative blood cultures. The diagnostic was non-bacterial thrombotic endocarditis related to cancer. The anticoagulant was changed to low molecular weight heparin, confirming the disappearance of vegetations.
\end{abstract}

Keywords: non-bacterial thrombotic endocarditis, marantic endocarditis, endometrial adenocarcinoma, deep vein thrombosis, apixaban

además como tratamientos crónicos toma valsartán y simvastatina.

A la exploración no se aprecia focalidad neurológica de vías largas, siendo la auscultación cardio-pulmonar y el resto de la exploración normales.

En pruebas complementarias destaca: hemoglobina $11,8 \mathrm{~g} /$ $\mathrm{dll}$, leucocitos $8,5 \times 10^{9} / \mathrm{L}$, plaquetas $222 \times 10^{9} / \mathrm{L}$, INR 1,5 , procalcitonina 0,7 ng/ml, PCR 6,6 mg/dl, glucosa $160 \mathrm{mg} / \mathrm{dl}$, creatinina $0,6 \mathrm{mg} / \mathrm{dl}$, con aclaramiento calculado de $47 \mathrm{ml} /$ min. En el sedimento urinario se evidencia leucocituria (16-20 leucocitos/campo) y bacterias escasas. La radiografía de tórax es normal. El ECG presenta un ritmo sinusal sin alteraciones significativas.

La tomografía computarizada craneal (TC) no evidencia patología aguda. Se ingresa, añadiendo al tratamiento amoxicilina/ ácido clavulánico vía parenteral. El urocultivo es negativo.

A las 48 horas de su ingreso se objetiva disfasia motora. Una nueva TC craneal demuestra un infarto isquémico agudo en región de arteria cerebral media izquierda.

Los estudios vasculares de troncos supra-aórticos y el holterECG no demuestran datos patológicos. Una ecocardiografía transtorácica detecta hipertrofia de ventrículo izquierdo, sin valvulopatías significativas ni datos de endocarditis. Existe elevación de CA 19.9 (180 U/mL) y Ca125 (184 U/mL).

Una TAC tóraco-abdómino-pélvica objetiva infartos en bazo y en riñón izquierdo, así como útero de aspecto nodular, con ocupación de cavidad endometrial, captación heterogénea de contraste y múltiples adenopatías retroperitoneales y en cadenas ilíacas (Figura 1). 


\begin{tabular}{|c|c|c|c|c|c|c|}
\hline REFERENCIA & EDAD & TIPO DE TUMOR & $\begin{array}{l}\text { VÁLVULA } \\
\text { AFECTADA }\end{array}$ & $\begin{array}{l}\text { MANIFESTACIONES } \\
\text { ISQUÉMICAS }\end{array}$ & TRATAMIENTO & $\begin{array}{l}\text { EVOLUCIÓN DE } \\
\text { VERRUGAS }\end{array}$ \\
\hline Glass J.P. 1993 & 42 & CARCINOMA DE CÉRVIX & Mitral & Cerebro & Ninguno & $\begin{array}{l}\text { Diagnosticadas } \\
\text { en autopsia }\end{array}$ \\
\hline $\begin{array}{l}\text { Numnum T.M., } \\
\text { et al. } 2006\end{array}$ & 38 & $\begin{array}{l}\text { ADENOCARCINOMA ENDOMETRIOIDE DE ÚTERO } \\
\text { ADENOCARCINOMA ENDOMETRIOIDE DE OVARIO }\end{array}$ & $\begin{array}{c}\text { Aórtica } \\
\text { Tricuspídea }\end{array}$ & $\begin{array}{l}\text { Riñón, Bazo, Cerebro, } \\
\text { Cerebelo, manos. }\end{array}$ & $\begin{array}{c}\text { Cirugía } \\
\text { Quimioterapia } \\
\text { Radioterapia }\end{array}$ & $\begin{array}{c}\text { Cirugía de } \\
\text { sustitución } \\
\text { valvular }\end{array}$ \\
\hline $\begin{array}{l}\text { Mir 0., et al. } \\
200\end{array}$ & 40 & TUMOR NEUROECTODÉRMICO DE MIOMETRIO & Aórtica & Riñón, Bazo, Cerebro & $\begin{array}{c}\text { Cirugía, } \\
\text { Quimioterapia. }\end{array}$ & $\begin{array}{c}\text { Cirugía de } \\
\text { sustitución } \\
\text { valvular }\end{array}$ \\
\hline $\begin{array}{l}\text { Ito S., et al. } \\
2013\end{array}$ & 60 & ADENOCARCINOMA ENDOMETRIOIDE DE ÚTERO & Mitral & Riñón, Bazo, Cerebro. & $\begin{array}{c}\text { Cirugía } \\
\text { Quimioterapia } \\
\text { Radioterapia }\end{array}$ & Desaparición \\
\hline $\begin{array}{c}\text { Grecu N., et al. } \\
2014\end{array}$ & 65 & ADENOCARCINOMA MUCINOSO DE ENDOCÉRVIX & Mitral & Cerebro, Cerebelo & No documentado & No documentada \\
\hline $\begin{array}{l}\text { Hottois E., et al. } \\
2014\end{array}$ & 47 & CARCINOMA DE CÉRVIX & Mitral & Cerebro, manos & $\begin{array}{l}\text { Quimioterapia } \\
\text { Radioterapia }\end{array}$ & No documentada \\
\hline $\begin{array}{l}\text { Erturk N.K., et } \\
\text { al. } 2015\end{array}$ & 56 & $\begin{array}{l}\text { ADENOCARCINOMA ENDOMETRIOIDE DE ÚTERO } \\
\text { ADENOCARCINOMA ENDOMETRIOIDE DE OVARIO }\end{array}$ & Mitral & Cerebro. & $\begin{array}{c}\text { Cirugía } \\
\text { Quimioterapia }\end{array}$ & No Documentada \\
\hline $\begin{array}{l}\text { Orfanelli T.,et al. } \\
2016\end{array}$ & 63 & $\begin{array}{l}\text { ADENOCARCINOMA ENDOMETRIOIDE DE ÚTERO } \\
\text { CARCINOMA PAPILAR SEROSO DE OVARIO }\end{array}$ & $\begin{array}{l}\text { Aórtica } \\
\text { Mitral }\end{array}$ & No Documentada & $\begin{array}{c}\text { Cirugía } \\
\text { Quimioterapia }\end{array}$ & Desaparición \\
\hline $\begin{array}{l}\text { Yokoyama S.,et } \\
\quad \text { al. } 2016\end{array}$ & 42 & ADENOCARCINOMA ENDOMETRIOIDE DE ÚTERO & Mitral & Cerebro. & $\begin{array}{c}\text { Cirugía } \\
\text { Quimioterapia }\end{array}$ & Desaparición \\
\hline Caso actual & 80 & ADENOCARCINOMA ENDOMETRIOIDE DE ÚTERO & $\begin{array}{l}\text { Aórtica } \\
\text { Mitral }\end{array}$ & Riñón, Bazo, Cerebro. & Ninguno & Desaparición \\
\hline
\end{tabular}

Se repite la ecocardiografía, vía transesofágica (ETE), a los 15 días del ingreso que demuestra: aurícula izquierda moderadamente dilatada, con orejuela libre de trombos; en la superficie auricular sobre ambos velos mitrales, presenta estructuras de un diámetro de 1,1 cm sugestivas de vegetaciones; en la válvula aórtica se evidencian estructuras compatibles con vegetaciones en cara ventricular de velo no coronario; existe insuficiencia mitral severa; no existen datos de shunt intracardíaco (Figura 2). Se extraen hemocultivos y serologías de Brucella, Bartonella, y Coxiella, suspendiendo administración de amoxicilina/ácido clavulánico, e iniciando tratamiento antibiótico con ceftriaxona a dosis de $2 \mathrm{~g}$ intravenosa (IV) cada 24 horas (durante 15 días), ampicilina $2 \mathrm{~g}$ IV cada 4 horas (7 días) y doxiciclina $100 \mathrm{mg}$ vía oral cada 12 horas (15 días).

Los hemocultivos iniciales y las serologías fueron negativos.

El estudio de trombofilia demuestra resultados negativos para anticoagulante lúpico, anticuerpos anticardiolipina, y anti-beta2-glicoproteína I.

Ante la ausencia de datos clínicos y analíticos que sugiriesen endocarditis infecciosa, y la alta sospecha de ETNB, se cambia apixaban por biosimilar de enoxaparina a dosis anticoagulantes ajustada a peso.

Se repiten los hemocultivos una semana después de la suspensión de los antibióticos, con resultados negativos.

Transcurridos de 18 días del cambio de tratamiento anticoagulante se realiza una nueva ETE, sin apreciarse datos significativos de verrugas, así como con mejoría de la insuficiencia mitral.
Se realiza biopsia de endometrio siendo diagnóstico de adenocarcinoma tipo endometriode. La paciente es valorada conjuntamente por los Servicios de Ginecología y Oncología, decidiéndose alta médica con tratamiento conservador y vigilancia clínica.

\section{DIAGNÓSTICO}

Síndrome de Trousseau: Trombosis venosa profunda y endocarditis trombótica no bacteriana relacionadas con cáncer endometrio.

\section{DISCUSIÓN}

La presencia de ETNB en pacientes con cáncer presenta datos contradictorios: mientras estudios ecográficos en pacientes vivos con cáncer se evidencia ETNB en un 19\% del grupo, los estudios post-mortem, sólo detectan lesiones de ETNB entre el $0,9-1,3 \%{ }^{3}$.

Las verrugas de la ETNB suelen ser pequeñas, estériles, compuestas de fibrina y plaquetas, friables y fácilmente embolizables. Se localizan de forma mayoritaria en la porción distal de válvulas, en la superficie auricular de la válvula mitral y la superficie ventricular de la válvula aórtica ${ }^{1,6}$.

Las manifestaciones clínicas de la ETNB dependen más de los fenómenos embólicos, sobre todo en bazo, riñón, y encéfalo, y menos de la disfunción valvular provocada por las verrugas ${ }^{1}$. El método más sensible para el diagnóstico de las verrugas es la ecocardiografía transesofágica'.

El manejo terapéutico de la ETNB relacionada con cáncer se debe basar en el tratamiento anticoagulante y en tratamiento del propio tumor. El tratamiento anticoagulante en 

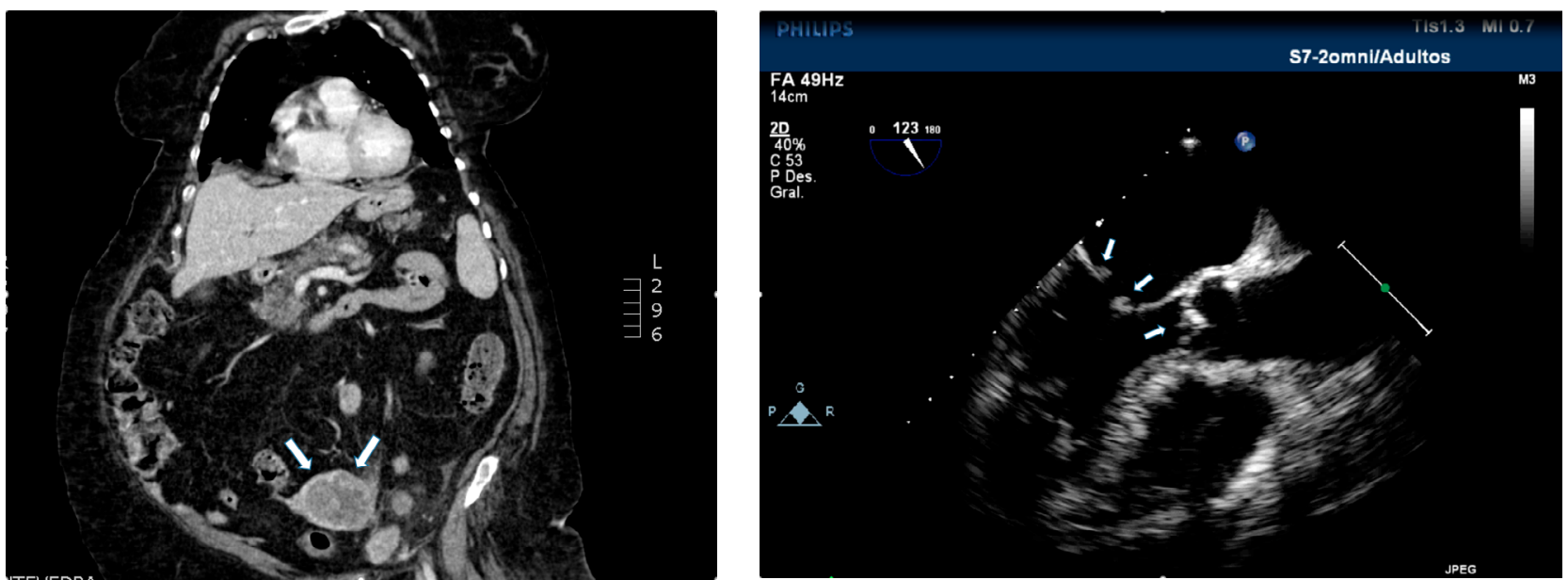

estos pacientes no ha sido definido, aunque se recomienda, bien heparina no fraccionada (HNF), o heparina de bajo peso molecular (HBPM)'.

En la TVP relacionada con el cáncer, las guías más recientes recomiendan tratamiento basado en anticoagulantes orales de acción directa. Se podría pensar en hacer extensible dicho tratamiento a pacientes con ETNB asociada al cáncer, pero nuestro caso junto con otros similares, ponen en evidencia la mala evolución clínica de estos pacientes tratados con anticoagulantes orales de acción directa (AOAD) ${ }^{6}$.

De los 9 casos publicados de ETNB y cáncer de útero (Tabla 1), la evolución clínica de las verrugas mejora y es documentada su desaparición con ecocardiografía en 3 pacientes. De los restantes, en tres no está documentada, dos son sometidos a cirugía cardíaca valvular sustitutiva, mientras que en uno se diagnostica la ETNB en la necropsia. En aquellos pacientes donde se evidencia la desaparición de las verrugas, todos reciben tratamiento anticoagulante con heparina, así como tratamiento oncológico basado en cirugía, quimioterapia, y radioterapia.

En nuestro paciente se evidencia la práctica desaparición de las verrugas sólo con tratamiento anticoagulante basado en HBPM. Creemos que el tratamiento con HBPM hizo disminuir significativamente el tamaño de las verrugas hasta su práctica desaparición.

\section{BIBLIOGRAFÍA}

1. el-Shami K, Griffiths E, Streiff M. Nonbacterial thrombotic endocarditis in cancer patients: pathogenesis, diagnosis, and treatment. Oncologist. 2007; 12(5):518-23.

2. Numnum TM, Leath CA 3rd, Straughn MJ Jr. Synchronous primary endometrial and ovarian carcinoma in a patient with marantic endocarditis. Obstet Gynecol. 2006 ;108(3 Pt 2):748-50.

3. Ito S, Yoshitomi H, Pak M, Kawahara H, Oshima T, Ito S, Watanabe N, Sato H, Adachi T, Takeda M, Sugamori T, Takahashi N, Endo A, Ishibashi T, Tanabe K. Trousseau syndrome with nonbacterial thrombotic endocarditis in a patient with uterine cancer. Intern Med. 2013; 52(12):1353-8.

4. Erturk NK, Erturk A, Basaran D, Ozgul N. Synchronous Ovarian and Endometrial Endometrioid Adenocarcinoma Presenting with Nonbacterial Thrombotic Endocarditis and Pulmonary Thromboembolism: Adenocarcinoma with Thrombotic Events. Case Rep Obstet Gynecol. 2015; 2015:825404.

5. Yokoyama S, Iwano H, Yamada S, Takeda M, Kaga S, Nakabachi M, Nishino H., Ichikawa A, Abe A, Okada K, Murai D, Hayashi T, Nishida M, Shibuya H, Kahata K, Shimizu C, Mikami T, Tsutsui H. Long-term echocardiographic evaluation of valvular lesions in a patient with nonbacterial thrombotic endocarditis associated with advanced uterine cancer. J Cardiol Cases. 2016 ;14(3):82-86.

6. Orfanelli T, Sultanik E, Shell R, Gibbon D. Nonbacterial thrombotic endocarditis: A rare manifestation of gynecologic cancer. Gynecol Oncol Rep. 2016; 17:72-4.

7. Glass J.P. The diagnosis and treatment of stroke in a patient with cancer: nonbacterial thrombotic endocarditis (NBTE): a case report and review Clinical Neurology and Neurosurgery; 1993; 95: 315-318.

8. Grecu N, Tiu C, Terecoasa E, Bajenaru 0. Endocarditis and stroke. Maedica (Buchar) 2014; 9(4):375-81.

9. Mir 0, Albiges L, Arkwright S, Duboc D, Massault PP, Tenenbaum F, Touzé E, Menasché P, Vacher-Lavenu MC, Goldwasser F, Alexandre J. Primitive neuroectodermal tumor of the myometrium with cardio-vascular symptoms. Gynecol Oncol. 2007;106(3):622-5.

10. Hottois E, Weichselbaum L, Guérisse F, Doriath V [Neurologic disorder complicating a cervical cancer]. Rev Med Brux. 2014 Mar-Apr;35(2):72-7.

11. Kearon C, AkI EA, Ornelas J, Blaivas A, Jimenez D, Bounameaux H, Huisman M, King CS, Morris TA, Sood N. Stevens SM, Vintch JRE, Wells P, Woller SC, Moores L. Antithrombotic Therapy for VTE Disease: CHEST Guideline and Expert Panel Report. Chest. 2016;149(2):315-352.

12. Mantovani F, Navazio A, Barbieri A, Boriani G. A first described case of cancerassociated non-bacterial thrombotic endocarditis in the era of direct oral anticoagulants. Thromb Res. 2017 ;149: 45-47. 